\title{
Internationalizing the University: A Case Study of a Canada-China Program
}

\author{
Huiqin Yang1, Barry Lesser ${ }^{2 *}$ \\ ${ }^{1}$ Shandong University of Finance and Economics, Jinan, China \\ ${ }^{2}$ Dalhousie University, Halifax, Canada \\ Email: ^barry.lesser@dal.ca
}

How to cite this paper: Yang, H. Q., \& Lesser, B. (2017). Internationalizing the University: A Case Study of a CanadaChina Program. Creative Education, 8, 359372 .

https://doi.org/10.4236/ce.2017.83028

Received: November 30, 2016

Accepted: March 10, 2017

Published: March 13, 2017

Copyright ( 92017 by authors and Scientific Research Publishing Inc. This work is licensed under the Creative Commons Attribution International License (CC BY 4.0).

http://creativecommons.org/licenses/by/4.0/ (c) (i) Open Access

\begin{abstract}
Internationalization has become a goal of most universities in the world and includes increased student mobility across borders. Goals behind greater student mobility are mixed, but whatever the goals are, all institutions should strive to try to maximize the net benefits for students. An economics program between Dalhousie University in Canada and Shandong University of Finance and Economics in China is used to examine how net benefits can be maximized. Amongst the conclusions reached by the paper, it is students who participate in exchange or study abroad programs that will experience an increase in intercultural competence, which is a major benefit of the experience. A second significant conclusion is that language is a crucial determinant of success when students must adopt a foreign language when they study abroad. The paper also shows that faculty engagement is crucial to success. Strictly top-down programs that originate and/or reside in the central administration are less likely to have, and less likely to be successful without faculty engagement. The paper concludes that the institutions must have a commitment to the program that goes beyond a simple short term profit maximization motive but ultimately, the success of any program depends on the students themselves. Other factors can defeat a given program, but only the students can ultimately make a program successful.
\end{abstract}

\section{Keywords}

Internationalization, International Student Mobility, Faculty Engagement, Intercultural Competence, Higher Education

\section{Introduction}

Increasingly, around the world, university students are engaging in study abroad programs of various kinds. Part of this is a reflection of a growing emphasis by 
many universities on so-called internationalization ${ }^{1}$, a concept which includes making an exposure to different cultures and countries a part of a student's educational experience. Exposure in this context can take many forms, ranging from the creation of new courses focused on other countries and/or other cultures, changing the content of existing courses to better reflect international dimensions of the subject matter, study abroad programs for your own students, recruitment of students from other countries to foster more diversity in the student body, etc. Part, also, is financially motivated in two respects for western universities, in particular, but some others as well; foreign students are an important source of new students to supplement the domestic supply of students, which in many of these countries is a declining number, and hence, constitute an important new revenue stream for western universities (Adnett, 2010); and for some developing countries, it is cheaper or more cost-effective, at least in the short run, to have students study abroad rather than add to domestic capacity in the educational sector ${ }^{2}$. Examples of countries that have particularly addressed this approach are interalia Saudi Arabia, Brazil and China.

Whether the growth in student mobility across borders is quality driven or financially driven ${ }^{3}$, the result is more students, particularly in western universities, who come from outside the country in which they are studying. On one hand, this creates a tremendous opportunity for increased diversity in the student body, with all the attendant benefits that potentially go with, or are created by, such diversity. On the other hand, it presents the challenge of how to ensure that this opportunity produces a net positive benefit for all students, regardless of where they come from. It is not sufficient to simply put people from different cultures in the same classroom to realize the potential benefits of such a step. It has to be done in a way that allows the students in question, both domestic students and foreign, not only to accept each other's presence but to take advantage of the greater diversity to enhance their own experience as students. Some of this is a function of the students, i.e., they have to see the potential benefits and take steps to realize these benefits for themselves. But part is also up to the institution, both in terms of providing support services that allow benefits to be realized and more simply, nurturing in their students a sense of why diversity is a good thing and an appreciation of the potential benefits of greater diversity ${ }^{4}$.

${ }^{1}$ For a broader discussion of aspects of internationalization, see, for example, Ilieva et al., 2014; Li \& Tu, 2016; Mohsin \& Zaman, 2014.

${ }^{2}$ This is discussed in OECD 2008. For an alternative view of this argument, i.e., the argument that getting students educated abroad is good for developing countries, see Adnett 2010.

${ }^{3}$ Mohsin and Zaman 2014 discussed four motivations for internationalization: political, economic, social/cultural and academic. Only the social/cultural and economic arguments are being addressed here; this does not mean that the others are not considered important but simply that they are not as relevant to the present discussion.

${ }^{4}$ Diversity in the context of internationalization can mean different things. It can relate to the influence of a multi-national group of students on the actual content of a course or to interactions between instructors and students or to interactions amongst students themselves. Any or all of these meanings may apply to our use of the term, although we clearly are less concerned with the issue of course content than others might be, largely because the program we are about to examine makes a deliberate point of assuring the students from abroad that they will receive/participate in the same courses as all other students at the institution. For a fuller discussion of the meaning of diversity see Ilieva et al., 2014. 
At the institutional level, there needs to be a recognition not only of the potential benefits that greater diversity can produce, but also of the potential costs of greater diversity. This point is fundamentally crucial to appreciate. There should be no doubt that there are potential costs that accompany greater diversity and these cannot and should not be ignored. The aim of the institution in these circumstances should be to maximize the benefits and minimize the costs. Elimination of all costs is probably not feasible, even if it might be possible, which is probably not the case. So minimization of costs is what we are left with. By the same token, we want the greatest net benefit, not only for the institution but more importantly, for the students ${ }^{5}$. This means not only minimizing costs but trying to maximize benefits in order to make net benefits as large as possible.

This paper uses a program between Dalhousie University (Dal) in Canada and Shandong University of Finance and Economics (SDUFE) in China as a case study for an analysis of how net benefits can be maximized or at least how costs and net benefits can be identified. In using this example, the reader is cautioned to be careful not to over-generalize from this one case. Some of the lessons of this single program may not apply to other programs involving other institutions and/or other countries. But hopefully, this example can be helpful in at least pointing out some things that should be addressed by any program of international cooperation in the post-secondary sector. A brief description of the Program is first provided, followed by a review of the questionnaire/survey process used and a discussion of some of the major points to emerge from the survey and a set of interviews with current students now at Dalhousie. Finally, some general conclusions are drawn, with the important qualification that care must be taken in drawing general conclusions from a single program.

\section{Program Description}

The Dalhousie University-Shandong University of Finance and Economics Joint Program in Economics started officially in 2008 when the first students were recruited to the Program in China. The Agreements governing the Program were signed by the two institutions in two stages, with the first agreement signed in 2006 and the second in 2007. The first official recruitment to the Program was in 2008 and the first students came to Dalhousie in $2009^{6}$.

Under the terms of the Program Agreement, SDUFE recruits students from high school and these students then have the option of spending four years at SDUFE or spending two years at SDUFE followed by two years at Dalhousie. If

${ }^{5}$ This is an important point. As Ilieva et al., 2014 point out, the financial motivation for internationalization, by itself, may not be concerned with other benefits of internationalization. This is unfortunately, too often the case. It is important to note, however, that the financial or economic benefits of internationalization and the social/cultural benefits, for example, are not mutually exclusive. In other words, the fact that a university tries to recruit more international students for economic reasons does not mean that they cannot and should not try to make the experience as meaningful as possible for both the foreign student recruits and the general student population of the university. ${ }^{6}$ The first students to come to Dalhousie were not in fact from the 2008 recruitment but rather seven students from the general student population at SDUFE to whom the Program was made available a year before the first students directly recruited to the Program from high school were able to come to Dalhousie. 
they choose the former, they receive a degree from SDUFE and a certificate of participation from Dalhousie. If they choose the latter, they receive a degree from both institutions. Students who spend four years at SDUFE receive eight courses taught by Dalhousie faculty or Dalhousie-recruited faculty, while those who spend two years at each institution get all Dalhousie-taught courses in their two years at Dalhousie and take three courses in their second year at SDUFE that are part of the eight Dalhousie-taught courses that the four-year SDUFE students do.

The Program is specific to the field of economics, so all students in the program, regardless of whether they spend time at Dalhousie or not, must have economics as their declared major. This point is important to appreciate in the context of this paper because some students end up in the Program because their parents think economics is a good field of study but the students may not in fact be particularly interested in the study of economics and/or either the students or their parents may choose this Program because they want a study-abroad experience and take this program because it offers this opportunity, even though the field of study may not be their first choice. Because much of what this study uses as "data" comes from a survey of students in the program, it is important to recognize that students may take the program for diverse reasons, some of which are unrelated to the field of study and where the field of study may negatively influence certain opinions about the program and its quality.

As noted above, the first recruitment of students directly to the Program took place in 2008. In that year, approximately 196 students were recruited. The following year, the recruitment was reduced, while SDUFE tried to decide how large it wanted the program to be. The decision made during the 2009-10 academic year was to go up to 250 and this number was achieved in 2010, 2011 and 2012. In 2013, the national Ministry of Education of China "advised" universities to pay attention to official quotas, which for the Dal-SDUFE Joint Program in Economics is 100 students per year. So in 2013, recruitment fell to $200^{7}$. The next year, the Ministry issued a written order regarding the observance of quotas and the number recruited in 2014 was 100. In 2014-2015, the program was one of several hundred reviewed by the Ministry and although the Program passed the review with no changes required, the over-quota numbers were noted and a penalty imposed limiting recruitment to 50 students in each of 2015 and 2016. For each cohort up to 2015, the number of students going to Dalhousie has been as shown in Table 1.

Generally speaking, the performance of the SDUFE students at Dalhousie has been excellent. The average GPA for these various cohorts has been above 3.0 in all years and generally is better than non-Program economics majors at Dalhousie $^{8}$.

${ }^{7}$ Permission for this, as for the previous excess numbers, was given by the provincial Bureau of Education. SDUFE was not willfully defying the government directive but dealt with Shandong Province for the permission, not the national ministry.

${ }^{8} \mathrm{An}$ interesting fact to note on performance is that twice in the last five years, the Dalhousie University Medal in Economics, awarded to the highest passing honour's student in the discipline in the graduating class for that year, has gone to one of the SDUFE students at Dalhousie. 
Table 1. SDUFE Students at Dalhousie, 2009/10-2015/16.

\begin{tabular}{ccc}
\hline Year & Number of New Students at Dalhousie & Number Graduating to Date \\
\hline $2009 / 10$ & 7 & 7 \\
$2010 / 11$ & 34 & 32 \\
$2011 / 12$ & 24 & 24 \\
$2012 / 13$ & 31 & 28 \\
$2013 / 14$ & 42 & 47 \\
$2014 / 15$ & 54 & 55 \\
$2015 / 16$ & 49 & N/A \\
\hline
\end{tabular}

\section{Survey Questionnaire}

The survey of students that was conducted between September 2015 and December 2015 involved a total of 80 respondents who completed both a written questionnaire and took part in a personal interview which provided an opportunity to expand on some of the survey questions. The number of participants reflected about $80 \%$ of the total population of students on hand at the time. Thus, participation is made up entirely of current students in either third year or fourth year of their undergraduate program of study. This means that all answers and opinions are short-term in nature in the sense that it is the reaction of students in the midst of the experience they are commenting on; it does not reflect the views of a more mature group several years after graduation able to look back on their experience and judge the long-term impact. Both perspectives are potentially valuable and have their place and at some point in the future, the authors hope to be able to offer some of the longer term perspective which the current study lacks. But that being said, let's turn our attention to seeing what the students told us.

A copy of the survey questionnaire is available on request. In what follows, there will not be any comprehensive summary of the overall responses to the entire questionnaire but rather, a selective discussion of some key points that emerge from a reading of the responses and interview notes.

\section{Degree of Difficulty}

It is often heard from students, before they go abroad, that they are concerned it will be too difficult. This, of course, can mean different things or more than one thing, depending on the individual. For some, it is a comment about their academic performance while for others it is about language and/or cultural adaptation. So one of the areas of question put to the students was the issue of difficulty and whether Dalhousie's curriculum was more difficult than SDUFE. Most students rated Dalhousie as more difficult than SDUFE although a few (5) rated Dalhousie as easier. Some noted that language was clearly a factor in this, i.e., Dalhousie was harder because it was all taught in English. Some suggested that differences in structure made it harder, not differences in content. And several felt that the Dalhousie instructors were stricter than their Chinese counterparts. On the question of pedagogical approach, there was a general consensus that Canadian professors are more flexible and want the students to participate in the 
class, whereas the Chinese instructors tend to follow the textbook quite rigorously and not to deviate from the prescribed lessons most of the time. Canada puts more emphasis on learning to think for oneself, while the Chinese approach is to learn a prescribed body of knowledge. The Canadian instructors put greater emphasis on learning throughout the semester while the Chinese approach is to study intensively for the final exam. Interestingly, several respondents noted that the greater flexibility and spontaneity in the Canadian approach (our words, not the students') actually made the Dalhousie courses easier in a sense because the work was spread out over the term rather than being focused just on the final exam.

One of the areas that were singled out for a lot of attention was the issue of interactive learning approaches and the interaction between student and professor as well as amongst the students themselves. The fact that Canadian instructors encourage questions and want students to interrupt if they have a question was generally seen as a good thing, as is the encouragement of students to talk to the professor before and after class or during office hours. This being said, many students noted that their major interaction in class was with other Chinese students-many were most likely to sit next to Chinese students and to talk only to Chinese students. Students recognize that the opportunity to talk with the professor and/or other students is there but they do not necessarily avail themselves of the opportunity.

Interestingly, on the question of the difficulty of understanding the language and following instructions, the average rating was 2.4, putting it between "a little bit difficult" and "fairly difficult". Only one student ranked the language as extremely difficult. But on the question of the main barriers posed by the course materials, language was the most commonly cited barrier, with about $75 \%$ of respondents including language as at least one of the barriers they faced regarding materials. In other words, language is a problem but not always that big a problem. The responses to these questions were not divided by year of study, however, and the authors would expect that students in their first year at Dalhousie would rank language as a bigger obstacle than students in second year. Fourth year students (i.e., students in their second year at Dalhousie), when asked what differences they had experienced between their first year at Dalhousie and their second year, singled out language as an area of improvement, confirming in this sense our expectations about which group sees language as the bigger problem.

On the question of differences from third year to fourth, one of the most common responses is that the students feel they have become more independent and more mature. Along with these changes, they also feel more "tolerant", "selfassured", more "open to change", "more confident".

\footnotetext{
${ }^{9}$ While it would be nice to think that these are all changes that Dalhousie has engendered in these students, the fact is that these are general effects of study abroad that one would expect to find in any group of foreign students at any western university. In other words, these effects are largely independent of Dalhousie, as much as we would hope that Dalhousie is reinforcing all of these tendencies and not curbing them in any way.
} 
Interaction with Other Students, Instructors, Citizens.

One of the differences noted by several students for year four versus year three of their program study is the greater ease of interacting with non-Chinese students as well as professors and TAs as the time at Dalhousie gets longer. Nonetheless, a large proportion of students acknowledged that most of their contact was with other Chinese students rather than non-Chinese students, although most fourth year students reported higher levels of interaction with non-Chinese students than the third year students. Given that the third year students were in their first semester at Dalhousie, this is not surprising. It is actually encouraging that so many had significant levels of interaction by their fourth year, although it is disappointing that approximately $10 \%$ of fourth year students still reported zero or less than $5 \%$ of interactions with non-Chinese students and close to $20 \%$ of respondents reported $20 \%$ or less of their interactions were with non-Chinese students.

A few students reported having non-Chinese roommates but the overwhelming majority have just Chinese roommates and moreover, more than $90 \%$ of respondents report speaking only Chinese with their roommates. This is an important point, although it is not easy to see a solution. Insofar as language continues to be a problem for students, even in their second year at Dalhousie, speaking English at home would be of some value. But living with mostly Chinese roommates does not encourage this practice, in fact it discourages it. Students are told that a) they should not just spend time with their friends from China who came to Dalhousie with them and b) to speak English as much as possible, even with one another. Neither of these things seems to be happening, however. Nor is it obvious how to get students to change this behavior given that it is perfectly understandable that they choose to behave the way they do.

The question of interaction with professors and TAs is another interesting point. Again, language is obviously part of the reluctance to engage with instructors or TAs, especially in the first semester at Dalhousie. Responses from fourth year students indicate that there is improvement in this area over time. That being said, it is also the case that some of this is a reflection of differences in the academic culture of Canada versus China. Chinese students in general are not encouraged by their teachers to ask questions in class or to offer comment on things the instructor says in class. Canadian academic culture, on the other hand, puts a great deal of emphasis on interactive learning and emphasizes teaching methodologies that encourage interactive learning. The fact that a large number of fourth year students reported a change in this respect in terms of their willingness to approach professors and/or the lessened pressure they experienced in doing so is a positive development if one subscribes to an interactive learning approach ${ }^{10}$.

One thing that almost every student agrees on is that Halifax is a friendly place and very few students have experienced any overt discrimination living in

${ }^{10}$ If one does not, however, the fact that students get "converted" after they are in Canada for a year may be seen as an unfortunate development. 
Halifax. While this would likely be the same almost anywhere in Canada, it is not at all a surprising response given the general reputation of Halifax ${ }^{11}$.

\section{Attitudes}

The question of whether people are friendly raises the more general issue of attitudes, which can be examined with respect to all parties in the discussion, including:

- The Chinese students in the Program

- Teachers from both institutions involved with the Program and/or with students in the program

- Administrative personnel at both institutions involved with the Program and/or the students in the Program

- Non-Chinese students majoring in Economics at Dalhousie

- English teachers at Dalhousie.

There may be other parties worth mentioning, but for the moment, at least, we will restrict ourselves to these five groups.

The first point to emphasize is that attitudes matter. If one approaches a study abroad option from a positive perspective, it is far more likely to be beneficial than if one starts with a negative attitude that sees only problems. This is obviously true for the students but it is also true for the other groups, especially the teachers, and is not always seen as an obvious truth by all of these groups. Take the case of Dalhousie faculty, as an example.

One of the important characteristics of the Dalhousie-SDUFE Program in Economics is that it is based in a single department on the Dalhousie side. The Department of Economics conceived of the program, negotiated the terms, both with SDUFE and internally within Dalhousie, and sees itself as "owning" the program. In other words, it has a proprietary interest in the program and its success. In this sense, the students in this program are very different than the students who simply show up in the Department's courses. The Department has a stake in all of its students, of course, but this particular group of SDUFE students stands apart because they serve a purpose for the department that is different than the other students majoring in Economics. The Department started the Program in the first place as a way to grow in a time of otherwise budget restraint. And as long as the Program serves that goal for the department, the students in the Program stand apart from other students in the Department. But there are other differences that arise as a consequence of the fact that the Department created the Program. The Department gets to decide who to accept, for example, which is something that normally departments have no say in. The Program does create challenges for the Department, it must be admitted. Classes

\footnotetext{
${ }^{11} \mathrm{Halifax}$ is a particularly friendly place and it is normal to hear visitors praise the friendliness of the people of Halifax. Halifax is a small community from the perspective of the number of people living there, while it also boasts restaurants, entertainment venues and other amenities greater than might normally be expected for a city of its size. Halifax is a definite advantage for Dalhousie versus many other Canadian universities.
} 
are all bigger, i.e., have more students. More teaching is done by part-time instructors because the number of courses and/or the number of sections of each course is greater and the greater use of part-timers means that the pressure on regular faculty increases in various ways, such as the relative burden of supervising honours students or even master's students or writing reference letters for students applying to graduate school. If one sees the program as simply more work-more marking, longer office hours, more reference letters, etc. then the program suffers. Students no longer feel good about the program and the faculty no longer feels good about the program. Everyone loses if this happens. This is a problem that is more likely to emerge when it is the central administration of (any) university that runs such a program.

Fortunately, this has not happened in this case. On the questionnaire, students were asked what suggestions they would make to their Dalhousie instructors to be better prepared to teach Chinese students. Some useful suggestions, like "speak slowly" and "write more clearly on the blackboard" were made. But a significant number responded that there was nothing to say, that "you guys did well", "they are very good" or "most professors are very kind to Chinese students". A large number of students had nothing to say on this question, suggesting that they are relatively satisfied with the faculty at Dalhousie. On a different question, having to do with whether students have difficulty understanding Dal professors whose native language is not English, only one respondent out of 79 ranked this problem as basically non-existent. Others ranked it somewhere between a bit of a problem and a significant problem, suggesting that in this respect, students would like to have a more uniform standard of English in the classroom. This, of course, is hard to achieve when you have a highly diverse department, as Dalhousie Economics does. The department has eight out of 17 full-time tenured or tenure track faculty whose native language is not English and who speak English with distinct accents. In spite of the fact that this may create some problems in the classroom for students whose first language is also not English, it is also important to recognize that this diversity at the faculty level is an important factor in explaining the tolerance and level of acceptance of diversity at the student level, which ultimately is at the heart of the success of a program such as the Dal-SDUFE Program in Economics ${ }^{12}$.

\section{Other Features}

One of the features of the Dal SDUFE Program in Economics is that it requires all students, regardless of the English proficiency test score they start with, to take a two semester ESL Workshop in their first two terms at Dalhousie. Students who have less than an IELTS of 6.0 (or equivalent) to start must take full-time English until they get to 6.0 in order to get into the workshop. But all students then take the workshop, even those who start with an IELTS of 6.5 or

\footnotetext{
${ }^{12}$ For an interesting discussion of so-called Faculty Engagement in Internationalization (FEI), see Li and $\mathrm{Tu}, 2016$. Also of interest on the role of faculty "buying into" internationalization is SpencerOatey, 2012.
} 
above. In order to accommodate the workshop in their academic course schedule, students take only three academic courses plus the workshop in their first semester at Dalhousie and four courses plus the workshop in their second semester. Students then take three additional courses in the summer intersession so they finish the first academic year at Dalhousie with 10 courses, which is a normal full-time course load, plus the English workshop ${ }^{13}$.

On the survey, when the students were asked about the workshop, most said that they find it useful and helpful. They also indicated that they liked English taught in Canada (at Dalhousie) more than what is taught in China (at SDUFE). The opinions are not unanimous, as some feel the workshop is not useful or is a waste of time, or has not helped their English. Interestingly, there seems to be no significant difference in the opinions of the third year students (first year at Dalhousie) and the fourth year students (second year at Dalhousie). Because the third year students when surveyed were just in the first semester of the workshop, they were making a judgment that might or might not stand up to completion of the course. The fact that the fourth year students still felt positive about the experience is therefore of some significance, although the fact that they are two different groups, rather than the same group being tracked over time, means that we cannot put too much emphasis on this point. It is also important to put opinions regarding the workshop into the proper context. English is seen by most students as the single greatest problem they face in studying at Dalhousie. Most students also feel that their English improves significantly over the time they are at Dalhousie. In this context it is easy to credit the workshop either too much or too little in terms of how much it adds to solving the language problem. It is nonetheless a fact that the workshop is a relatively unique feature of the Dal-SDUFE Program in Economics. And insofar as language is seen as the biggest problem facing the students, anything that contributes to easing this problem is good.

Another feature of the Program worth noting is the special support services that the Program offers to the students in the Program. First, there is a full-time, Mandarin-speaking staff person employed by the program who serves as an advisor on both academic matters and personal ones for the students. Having a full-time staff person dedicated to a particular group of students is not usual in a Canadian university but it serves a very valuable purpose in this particular program. Having a person who can communicate with the students and their parents in their own language makes it even more unusual and a major plus for this Program. Second, a student mentor program that pairs Canadian or non-Chinese students with the new Chinese students each year helps the Program students meet non-Chinese peers, gives another opportunity for them to speak

${ }^{13}$ The normal entrance requirement for English proficiency at Dalhousie is IELTS 6.5 (or TOEFL iBt of 90). However, it is also allowed that students with IELTS 6.0 can begin their studies on aparttime basis while also taking an approved ESL program. This is the basis for the SDUFE Program proceeding as it has, with the workshop constituting an "approved" ESL program and three courses in the first semester along with the workshop representing a part-time academic role. 
English, and helps them become more socially integrated ${ }^{14}$. Third, a special social budget for the Program allows social events that include all students in the Department and this gives students an opportunity to meet other students in a non-academic context while also giving the non-Chinese students in Economics an opportunity to interact with the program students socially, which benefits all the students, Program and non-Program alike.

The mixing of the Program students and the non-Program students is an important point to emphasize. The Program students are fully integrated with other students majoring in Economics at Dalhousie. There is nothing different about their Program with the single exception of the ESL Workshop course, which is the only course that is exclusively for the Program students. The degree they get is the same as any other Economics student at Dalhousie gets. The courses are all shared with other Economics students. Some of these courses are dominated by Chinese students not because they are reserved for Chinese students but because there are so many Chinese students that they are a majority in some courses. But the students are not purposefully segregated or given sub-optimal teachers or sub-optimal courses, which, unfortunately, is sometimes the case at some western universities who offer special programs for particular groups of foreign students.

Two final features of the Program deserve comment. First, it is especially noteworthy that a very high proportion of the Program students go on to postgraduate study in North America or the UK after completion of their bachelor degree at Dalhousie. In some years, in excess of $70 \%$ of the students have gone to a master's program in Canada or the US or the UK. This is remarkable for two reasons: 1) it indicates how well the students do at Dalhousie that so many qualify for graduate school and 2) it is well above the average for the Department in terms of the number of graduates who go on to post-graduate study. On the questionnaire, when students were asked if they hoped to go to graduate school, 7 out of 79 said no or did not answer; the rest said yes. In other words, $91 \%$ of the students surveyed answered yes to this question. And when they were asked if being at Dalhousie would improve their chances of getting into graduate school, 55 out of 79 , or $70 \%$ said yes ${ }^{15}$.

\footnotetext{
${ }^{14} \mathrm{An}$ interesting by-product of the mentor program is that many of the Program students volunteer to serve as mentors in their second year at Dalhousie as a way to help the new cohort of Program students just arrived. The problem this poses is that the mentor program is intended to serve not only as a form of assistance and support for new students but also to bring Chinese and non-Chinese students together. This latter purpose is not being served if Program students mentor Program students. The way this is dealt with is to assign the Chinese Program student volunteers as mentors to the mentors, so they get to participate but they do not displace the non-Chinese students taking part in the mentoring program.

${ }^{15}$ The perception that being at Dalhousie improves the chances of being accepted to a good graduate school in North America is held by the students in part based on the experience of past student cohorts in the Program, i.e., the students in a given year know that students in the past had a high acceptance rate and so think they will do better as well. In fact, it is almost certainly true that being at Dalhousie helps when applying to graduate school, not because Dalhousie is a better university than SDUFE but because admissions officers at Canadian, US or UK universities are more likely to know Dalhousie and to have some appreciation of the standards that Dalhousie represents. By contrast, many admissions officers at these same institutions may never have heard of SDUFE and know nothing about its standards. A Dalhousie transcript therefore is not only helpful in a direct sense but it also helps put the SDUFE transcript for the students' first two years of university in better context, allowing it to be more properly weighted in the decision on admission.
} 
The second final observation has to do with the partner university. As a special approval program sanctioned by the Ministry of Education of China, the Dal-SDUFE Program in Economics has its own recruitment quota and runs independent of the other academic programs at SDUFE. This is typical of how such programs are usually organized on the Chinese partner side. But what SDUFE has done, which is not typical of all Chinese universities with such programs, is to integrate this program with its regular academic programs. This means that the School of Economics at SDUFE, which offers the Bachelor of Economics degree program for regular students of economics at SDUFE, is also an integral part of the Joint Program, even though another unit of the university has administrative responsibility for the Program. It is not uncommon for such special programs to use part-time teachers/instructors and for there to be no link between such programs and the rest of the university. This is not true for SDUFE and this has helped the program enormously both in terms of the quality of teachers at SDUFE, the preparation of students coming to Dalhousie, the preparation of students who stay at SDUFE for the courses they must take taught by Dalhousie instructors and for the overall reputation of the Program within SDUFE.

When students were asked what suggestions they would make to their Chinese instructors so the students were better prepared for study in Canada, about $30 \%$ of respondents had no comment or noted that they had nothing to suggest. In other words, $30 \%$ were relatively satisfied with things as they are. For the others, many suggestions related to specific information about living abroad, or language skills, things that are not necessarily the job of the Chinese instructors. Some had to do with general pedagogical differences between Canadian teaching methods and Chinese, which are legitimate areas for debate but not necessarily a criticism of either group. And some had to do with institutional practices in China, including such things as the relative weight of the final exam in the overall grade for a course. In other words, very few if any of the comments reflect a dissatisfaction with specific instructors or the overall quality of the Chinese instructors, which in turn, we would argue, reflects on the way in which SDUFE has organized the teaching resources committed to the Program.

\section{Conclusion}

Overall, as noted earlier, care must be taken in drawing general conclusions from this program's experience. Nonetheless, some conclusions can be offered that are at least confirmed by this program. These are as follows:

1) Students who study abroad are positively oriented for embracing change.

2) By choosing to live and study in a culture other than their own, these students develop an intercultural competence. This is defined by Kim, 2009 as "the capacity of an individual to enact behaviors and activities that foster cooperative relationships with culturally (or ethnically) dissimilar to others".

3) Language is a crucial determinant of the relative success of foreign study programs for any program which is not a language program per se. This not only 
means that students must be well-prepared in the foreign language before entering the foreign university, but that special efforts may be called for after entering to develop and reinforce language proficiency. The ESL Workshop in the Dal-SDUFE Program serves this purpose and the specially designed structure of the first year's courses (3-4-3 over ten months) versus the conventional full course load of 5-5 for two semesters allows this language reinforcement to occur.

4) The idea of Faculty Engagement in Internationalization (FEI) as outlined by $\mathrm{Li}$ and $\mathrm{Tu}$ (2016) is an important one. Too often, foreign student study programs fail to live up to their potential because the promotion and recruitment are carried out by centralized offices of the university and the students are then simply "dumped" into the general student population. In such cases, individual faculty who teach these students see nothing special about them and make no attempt to change what they do to accommodate these students. Central support services may exist, but it is up to the student to decide whether to access these services and those students who don't, are basically lost in the crowd ${ }^{16}$. Faculty in general will not buy into foreign student study programs or other internationalization objectives of the institution merely because they are asked/told to do so by senior officials. As Li and Tu (2016) pointed out, faculty must buy in on a personal basis if you want them to embrace the program and the students. At Dalhousie this has happened because the Department has not only initiated the Program, but also because they have been allowed to retain control of the Program in most respects and because they benefit directly from the Program. The special services that students in the Program enjoy have been/are crucial to the success of the Program.

5) The commitment of both institutions to the Program has been key to its success. Although the main objective of the Program for both sides is financial, both sides have been willing to invest in making the Program a relative success academically, even when this means the sacrifice of short term profits. Universities that are concerned only with maximizing short-term profit not only are less likely to be as successful but are also quite likely to see long term profits falling, because the reputation of the Program fails to be maintained over time ${ }^{17}$.

6) Ultimately, it is the students who make a Program successful. Institutions can support the Program in various ways that matter; faculty need to buy into the Program and the whole concept of internationalization; attention to language matters a great deal. But all of these are things that if not done right, they can deny success to a Program, and by themselves, they do not/cannot make a Program succeed. Only the students can ultimately define success and the

\footnotetext{
${ }^{16}$ In different words, most central programs are passive in the sense that they wait for the students to come to them and are not willing or not able to seek out the students to prevent problems versus responding to problems after they emerge and if they are brought to them.

${ }^{17}$ The Dal-SDUFE Joint Program in Economics has become a well-recognized "brand" in Shandong Province. Interalia, this has meant a higher Gaokao (university entrance exam) standard for the Program than for any other Joint Program in Shandong Province.
} 
Dalhousie-Shandong University Joint Program in Economics has been successful primarily because of its students. The high proportion of students who go on the graduate school in North America or the UK following graduation is indicative of this.

\section{References}

Adnett, N. (2010). The Growth of International Students and Economic Development: Friends or Foes? Journal of Education Policy, 25, 625-637. https://doi.org/10.1080/02680931003782827

Ilieva, R., Beck, K., \& Waterstone, B. (2014). Towards Sustainable Internationalization of Higher Education. Higher Education, 68, 875-889. https://doi.org/10.1007/s10734-014-9749-6

Kim, Y. (2009). The Identity Factor in Intercultural Competence. In D. K. Deardorff (Ed.), The Sage Handbook of Intercultural Competence (pp. 53-65). Thousand Oaks, CA: Sage.

Li, B. H., \& Tu, Y. J. (2016). Motivations of Faculty Engagement in Internationalization: A Survey in China. Higher Education, 71, 81-96. https://doi.org/10.1007/s10734-015-9890-x

Mohsin, A., \& Zaman, K. (2014). Internationalization of Universities: Emerging Trends, Challenges and Opportunities. Journal of Economic Information, 3, 1-21.

OECD (2008). Education at a Glance 2008. Paris: OECD.

Spencer-Oatey, H. (2012). Maximizing the Benefits of International Education Collaborations: Managing Interaction Processes. Journal of Studies in International Education, 17, 244-261. https://doi.org/10.1177/1028315312454545

\section{Submit or recommend next manuscript to SCIRP and we will provide best} service for you:

Accepting pre-submission inquiries through Email, Facebook, LinkedIn, Twitter, etc. A wide selection of journals (inclusive of 9 subjects, more than 200 journals)

Providing 24-hour high-quality service

User-friendly online submission system

Fair and swift peer-review system

Efficient typesetting and proofreading procedure

Display of the result of downloads and visits, as well as the number of cited articles

Maximum dissemination of your research work

Submit your manuscript at: http://papersubmission.scirp.org/

Or contact ce@scirp.org 